THEORIA ET HISTORIA SCIENTIARUM, VOL. VI, $\mathrm{N}^{\circ} 1$

Ed. Nicolas Copernicus University 2002

\author{
Charles Forceville
}

\title{
Further thoughts on delimiting pictorial metaphor
}

\section{Introduction}

Since Ortony's (1979) Metaphor and Thought and Lakoff and Johnson's no less Jifluential Metaphors We Live By (1980), the cognitive approach has become the dominant paradigm in metaphor studies. Its basic tenet is that metaphors are not primarily a characteristic of language; they first and foremost belong to the realm of thinking. The cognitivist paradigm presupposes that while most metaphors manifest themselves verbally, it would be a mistake to equate verbal "inifestations of metaphor with their cognitive originals. Thus, conceptual metaphors may assume different linguistic guises; "he attacked every weak point in my argument" and "his criticisms were right on target" both derive from the under lying metaphor ARGUMENT IS WAR (Lakoff and Johnson 1980: 4). Explorations of how conceptual metaphors may appear in language (for two

recrent studies see Goatley (1997) and Cameron and Low (1999)) and, conversely, of how verbal metaphors may be signposts to guide us to their conceptual sources have characterized a substantial part of the study of metaphor over the past two decades, but another entailment of the cognitivist approach has hitherto received short shrift. If metaphor is primarily a cognitive affair, and metaphors on the verbal level are not simply reduplications of metaphors on the cognitive level, pervasiveness metaphors need not be verbal in nature. The investigation of non-verbal and partly-verbal metaphors is necessary, both for their intrinsic interest and for the further development and testing of the cognitivist paradigm. Given the pervasiveness of visual representations in contemporary society, it is, unsurprisingly, primarily pictorial metaphor that has begun to attract the attention of scholars interested in non-verbal metaphor (e.g., see McNeill (1992) for observations on metaphor in gestures). 
In the interests of a healthy and more complete theory of what I call "pictorial metaphor" but which is also referred to as "visual metaphor" its nature must be explored in more detail. Since theorizing about pictorial metaphor has hardly got off the ground, it is specifically important to trace how pictorial metaphor relates to its parent conceptual metaphor and to its far more well-known sister verbal metaphor. After all, the justification for calling a certain phenomenon "pictorial metaphor" must reside in that phenomenon's strong family resemblance to uncontroversial examples of metaphor - which in practice hitherto means "verbal metaphor". In this article I want to point out and briefly discuss a number of issues pertaining to pictorial metaphor. Given the current state of affairs, there will be more questions than answers, but if the questions contribute anything to a further delimitation of pictorial metaphor, I shall be well satisfied. Let me begin by presenting in a nutshell the basic tenets from my own work on pictorial metaphor in advertising (Forceville 1996, 2000) as well as attempt to generalize some of these tenets. This will then provide a starting point for the limitations that study of the field has - some of them inherent in the corpus material chosen - which subsequently will facilitate thinking about the directions further research could take.

\section{Assumptions about pictorial metaphor in Forceville (1996)}

Lakoff and Johnson's observation that "the essence of metaphor is understanding and experiencing one kind of thing in terms of another" (1980: 5) should hold by definition for anything that deserves the name of pictorial metaphor. Their description usefully draws attention to the mental work that is done, or is to be done, by the recipient of the metaphor. A metaphor is thus not necessarily recognizable from unambiguous "text-inherent" cues; a metaphor must be construed as such. Secondly, the description mentions "kinds" of things. A kind, I take it, is a category in the sense of Lakoff (1987), which presupposes among other things that categories have more as well as less prototypical members. Moreover, while most categories that humans use in their daily lives are fairly stable ones, categories can also be conceptualized on an ad-hoc basis for very specific (and temporary) purposes. A category is amenable, if circumstances so demand, to further subdivision in such a way that two "things" that initially belonged to the same category end up being in different categories, so that they can now be used as target ("one kind of thing ${ }^{11}$ ) and source ("another kind of thing") of a metaphor. In her discussion of "semantic fields" - which are also categories - Kittay makes the same point:

We should individuate to the degree that is relevant to us. We may wish to individuate forests, trees within a forests, branches on a tree, leaves on a branch, or the cells 
which comprise the leaves of a tree. At what point we individuate these nested entities depends on our purposes (1987: 176).

While studies of verbal metaphor tend to concentrate on the issue of interpretation, that is, of deciding which features are to be mapped from source to target, and assessing how this mapping process affects the now metaphorically changed target, this process in fact presupposes two prior questions which are seldom made explicit. The first is: which are the two "things" involved in the so-called metaphor? And the second is: which of the two "things" is the metaphor's tenor (topic, target) and which is the metaphor's vehicle (source) $?^{1}$ The reason for the neglect of these two questions is, I suspect, twofold. One part of the explanation is that until recently most examples of verbal metaphors discussed in studies were either made up by the authors themselves or selected precisely because of the transparency of the tenor-vehicle distribution, so that there was insufficient awareness that this could be a problem at all. Another part of the explanation is that in paradigmatic verbal metaphors (at least in English!) tenors typically come before vehicles, and the two are usually linked by "is," or, in the simile variant, by "is like" ("surgeons are (like) butchers," "butchers are (like) surgeons") - and if not, grammar disambiguates the two ("butchers, that's what surgeons are!"). A picture, however, does not seem to impose a self-evident "linear-reading" order. Aumont mentions experiments on testing people's eye movements when they look at pictures, revealing

the complete absence of any regularity in the sequence of fixation points. There is no systematic reading of the image from top to bottom or from left to right, there is no visual plan. ...The only repeatedly obtained result is that... [eye] movements are influenced by the introduction of specific instructions to the viewer, which confirms that an informed gaze moves differently within the field of exploration (1997: 39).

Moreover, a picture has no visual signal equivalent to the verbal "is/is like". Finally, since a picture often contains many different "kinds of things" that are candidates for either tenor or vehicle positions in what could potentially be understood as a metaphor, it must first of all be decided which two elements ${ }^{2}$ might interact metaphorically, and subsequently which of them is the target and which of them the source. Thus, if something is to qualify as a pictorial metaphor - or indeed any other type of metaphor - the following three questions must be capable of being answered:

${ }^{1}$ In this article I will use the labels "tenor" and "vehicle". Tenor and vehicle will be taken as the named parts of the target and source domains, respectively, in the verbalizations of a metaphor.

${ }^{2}$ In this article I will not address the possibility of a pictorial metaphor containing more than two terms. 
(1) which are the two terms of the metaphor?

(2) which of the two terms is the metaphor's tenor/target, and which is the metaphor's vehicle/source?

(3) which feature(s) is/are mapped from vehicle to tenor?

If we want to identify a pictorial metaphor as such and to analyze it, being able to answer these questions should suffice; if we want to probe further into the nature of pictorial metaphor in general we might in each case complicate the question by adding "and how do we know"?

My first attempts at delineating pictorial metaphor pertained to Surrealist works of art, but while the examples, from paintings and collages by Magritte, Ernst, Dali and others, allowed a clear answer to the first question, the second raised greater problems, inasmuch as frequently a case could be made for A IS B as well as for B IS A. For instance, in Magritte's “L'explication" a table, situated against a mountainous background and a sky, contains a carrot, a wine bottle, and a hybrid object that is half-carrot and half-bottle (Forceville 1988: 155). Neither the pictorial context, nor the title gives a hint as to the dominance of either the bottle or the carrot, so that BOTTLE IS CARROT and CARROT IS BOTTLE are equally plausible (or implausible) renderings of the hybrid object. If a metaphor were at stake here, it would thus allow reversibility of tenor and vehicle. Since "irreversibility of tenor and vehicle" is a crucial aspect of metaphor in the cognitivist paradigm (e.g., Lakoff and Turner 1989: 132 3), I abandoned Surrealist work as, at the least, non-central to the project of developing a model of pictorial metaphor and concentrated on advertising instead. Since advertising, unlike Surrealist art, has a clear-cut if banal intention, namely to sell a product or service, advertising proved a better corpus. One of the metaphorical terms identified is identical with, or metonymically related to, the product, and this is the tenor of the metaphor. The other term, the vehicle, could be anything. The features mapped from vehicle to tenor are positive ones, emphasizing one or more qualities of the product. All of this was discussed in terms of Sperber \& Wilson's (1986/1995) Relevance Theory.

Four main types of pictorial metaphor were distinguished. The first type of metaphor (metaphors with one pictorially present term, or MP Is) depicts one term visually, while the nature of the other term is to be inferred from the pictorial context. An example is an advertisement for shoes which pictures a man's torso, clothed in shirt and formal jacket, while the expected TIE has been replaced by a (pictorial) SHOE. The only clue of the vehicle's identity (TIE), is the pictorial context (shirt, jacket). Potential mappings to the tenor SHOE include the TIE's

${ }^{3}$ In Forceville (1995) I argue that Lakoff and Turner are wrong to attribute the reversibility of tenor and vehicle to Black's interaction theory, as they do in the passage referred to. 
connotation of beauty, the expression of its owner's personal taste, and its proof that the wearer is aware of dressing etiquette. The second type (metaphors with two pictorially present terms, or MP2s) consists of hybrids, in which parts of both terms are visually represented (as in the bottle-carrot or carrot-bottle in Magritte's "L'explication"). An example is an ad, issued by the Dutch ministry of economic affairs in 1976, warning against the depletion of natural resources. It shows a hybrid of a globe and a burning candle, the feature mapped from candle to globe verbalizable as "using non-renewable energy". Pictorial similes, the third type, represent both terms in juxtaposed fashion, that is, in their entirety. An example is the juxtaposition of a watch and a butterfly, the verbal slogan "the profile of elegance" suggesting the nature of the feature to be mapped. The fourth type, verbo-pictorial metaphors (VPMs), presents one term pictorially and the other verbally. A billboard promoting the Amsterdam zoo Artis may serve as an example: a slightly smiling orang-utan is headed by the text "Mona Lisa", giving rise to the metaphor ORANG-UTAN IS MONA LISA 4 .

It is to be noticed that the last sub-type, verbo-pictorial metaphor, is no longer properly a pictorial metaphor, since it straddles the media of pictures and language. Indeed, the two "kinds of things" need not manifest themselves in the same medium. This is important, for it means that a metaphorical term (whether a tenor or a vehicle) can in principle occur in a wide variety of modes - as long as the following conditions are fulfilled:

(1) it is accessible to at least one of the senses;

(2) it is conceptualizable as a metaphorical term in the first place, that is, as a tenor or a vehicle (this also entails, of course, that it must be linkable with its complementary term - either vehicle or tenor);

(3) the term to be construed as vehicle evokes connotations that can be mapped onto the tenor in a relevant manner.

If this is accepted, the four subtypes can be seen as specimens of more general subcategories of metaphor, which can accommodate yet to be investigated types:

(Type A) MPls would then exemplify metaphors in which one of the terms is explicitly represented in a specific medium, while the other term is not explicitly represented but decisively "cued" by contextual factors in the same medium (pictorial context in a pictorial metaphor, verbal context in a verbal metaphor).

(Type B) MP2s would then exemplify metaphors in which both terms, represented in the same medium, have merged into a hybrid, which thus retains features of both.

(Type C) Pictorial similes would then exemplify metaphors in which both terms, represented in the same medium, are juxtaposed in their entirety.

\footnotetext{
${ }^{4}$ All examples, and many others, are discussed at greater length in Forceville (1996).
} 
(Type D) Verbo-pictorial metaphors would then exemplify metaphors in which the two terms are represented in different media.

It seems implausible to suppose that all variants are possible with all senses. For instance, whereas it may be possible to juxtapose (type C) two smells metaphorically, and to make a smell interact with a phenomenon in another medium (type D), or possibly even to merge two smells (type B) it seems unlikely that a smell "context" can cue a smell (type A). Furthermore it might well appear necessary to distinguish yet other types of non-verbal metaphor, while borders between types are sometimes fuzzy. But all this is virtually unexplored territory. Let me return to the three basic questions I have called essential for the analysis and interpretation of metaphor and discuss them with reference to its pictorial variant.

\section{Reflections on the question which are the two terms of the pictorial metaphor}

Clearly, the question which are the two terms of the pictorial metaphor is proleptic in the sense that it presupposes the presence of a pictorial metaphor in the first place. And if irreversibility of tenor and vehicle is a defining property, if not of all metaphors then at least of prototypical ones, then this question can hardly be separated from the question which of a metaphor's two terms is its tenor and which its vehicle. As Black (1979: 36) suggests, no failsafe procedure exists to identify a metaphor, but there certainly are clues to alert one to its presence. No doubt "salient identification" of two disparate elements characterizes a majority of phenomena that deserve the label "pictorial metaphor". The "disparateness" refers to the circumstance that the elements, in a given context, belong to different categories, resulting in a category clash. The "identification" refers to the circumstance that a metaphor should always be rephrasable as an A IS B formula, as the numerous examples in Lakoff and Johnson (1980) make clear. This criterion of rephrasability, however, must be applied thoughtfully and carefully. First, we should remember that while the manifestations of metaphor often already display this format, this is not necessary. The verbal metaphor "John is a sheep" reflects the cognitive metaphor JOHN IS A SHEEP, but so does "John bleated". In the latter case, it is not difficult to relate the manifestation to its conceptual origin. In other cases it may be slightly more difficult to perform this task:

[Norman] possessed the most hairless torso she'd ever seen on a post-pubescent male. His body had a plucked, waxed, defoliated look, as if it only remained to baste him in melted butter, and pop him into the oven at gas mark 6 with an apple in his mouth (Zahavi, 1993: 104). 
Here we must draw on our knowledge of the world of cuisine that it is usually pigs which are prepared in this way to recover the metaphor NORMAN IS A PIG. But while in this example it is fairly unproblematic to arrive at the A IS B format, the naming of the $\mathrm{A}$ and $\mathrm{B}$ term is not always self-evident. From the line "...the sun gnaws the night's bone/ down through the meat and gristle" in John Ridland's "Elegy for My Aunt" (quoted in Kennedy \& Gioia (1998: 124)), we can derive SUN IS ANIMAL-WITH-TEETH (and NIGHT IS BONE). But the "gnawing sun" example can also be verbalized as SHINING IS GNAWING, in which the focus is on the merciless activity of the sun rather than on its identity. This last example teaches an important lesson: construing the conceptual level of a metaphor is sometimes possible in more than one way, that is, different verbalizations of the metaphor (with ensuing different emphases in interpretation) may be valid. Sometimes it may even be impossible to come up with anything but a rather wrought, inelegant A IS B verbalization of a metaphor. Particularly when it is not nouns but verbs that are metaphorized, it may be difficult to come up with an unequivocal A IS B verbalization. Consider the first stanza of Carole Satyamurti's (1996) poem, "Spring offensive":

May's the month for optimistic acts: seedlings

- pansies, antirrhinums, salvias - bedded in,

gauche first-day-at-schoolers.

Your thumbs have blessed them, inner eye furnishing

dowdy beds with dazzling coverlets.

The word "blessed" in all likelihood is supposed to be taken metaphorically: we can construe the metaphor as, say, PRESSING-THE-EARTH-ON-SEED- LINGS-WHILEPLANTING-THEM IS (LIKE) BLESSING SEEDLINGS. This verbalization is somewhat awkward - probably because tenor and vehicle involve verbs rather than nouns. But we should not confuse the difficulty of verbalizing a metaphor in terms of the A IS B formula with doubting that establishing such an identity is possible.

When this awareness is applied to pictorial metaphors, it is clear that at least most examples of the first three types (that is, contextual, hybrid, and simile types) of pictorial metaphors can fairly easily be related to their conceptual A IS B parent, since for the metaphors to be depictable, they must preferably combine a tenor and a vehicle that are concrete. That is, pictorial metaphors often connect objects or persons - phenomena that in language are embodied in nouns. Hence many pictorial metaphors are "naturally" amenable to the A IS B formula. This does not mean, of course, that pictures have no means to metaphorize actions (in language rendered through verbs). Probably it is relatively difficult to render an action metaphorically in a static picture. But let us try to imagine how a director could visualize the Ridland metaphor mentioned above in a film: she could, for 
instance, show the sun shining in a cruelly hot manner (say with shimmering air, on tired people) in a desert, and then cut to a shot where a dog hungrily chews on a bone. If the director is worth her salt, she would find ways to make the crosscutting salient via camera angle, lighting, setting, corresponding colours, or any other from a host of stylistic options. The observation I want to make is that, given the context of our imaginary film, it may be more pertinent to verbalize the metaphor as SHINING IS GNAWING than as SUN IS DOG. If the point to be made in the scene or the film is that people suffer heat much like bones "suffer" being gnawed by dogs, the sun is not really of any intrinsic interest. Perhaps BEING CRUELLY EXPOSED TO HEAT IS BEING CHEWED-ON- BY-A-DOG catches the metaphor even better. In short, even though it should always be possible in a metaphor to recover a conceptual A IS B level, the rationale of the metaphor may not at all be captured in an easy verbalization on that level.

Recall the ORANG-UTAN IS MONA LISA example mentioned above for another illustration of this point. Apart from the text "Mona Lisa" the billboard contains the line "Live in Artis", so that the message of the metaphor is arguably that we should visit the zoo because it boasts this beautifully smiling "live" monkey, rather than go to faraway Paris to admire a mere painting. Again, that is, the metaphor could also be rendered as VISITING ARTIS IS LIKE VISITING THE LOUVRE (but in fact it is even more fun!), thereby focusing on two actions that are metonymically related to the original tenor and vehicle rather than on the tenor and vehicle themselves. Indeed, this verbalization reflects the objectives of the Artis billboard more accurately, since attracting (more) visitors is undoubtedly the primary goal of the billboard. Actually, some sort of action is almost always implied in any metaphor's NOUN PHRASE A IS NOUN PHRASE B formula. Interpreting a metaphor means envisaging what sort of activities that can be done with, to, by etc. the vehicle can be mapped onto the tenor. While it is useful to recapture the underlying A IS B formula for analytic purposes, it may be misleading to remain focused on that level. The A IS B is perhaps no more than a convenient "shorthand" to urge the audience to start exploring the target and source domains to which the tenor and the vehicle belong - and particularly the actions that exploring the domains enable one to perform. The limited use of the A IS B format can be gauged from the fact that in a different situation, the same metaphor could have been used to emphasize different connotations of the "Mona Lisa", for instance its financial value. Thus an animal rights organization, let us imagine, might want to warn us that orang-utans run the risk of becoming extinct, and by coupling one specimen with the "Mona Lisa" remind us of the species' "priceless" value. The metaphor could be captured as ORANG-UTAN IS MONA LISA just as in the first instance, but the metaphor's point would be a very different one. 
This brings us to another important factor besides "salience" for the identification of metaphor, and that is "relevance". Salient identification guides our attention, but if we see no reason to construe one of the elements in terms of the other, there will be no metaphor. In pictures, numerous things can be saliently juxtaposed, or even physically merged, without resulting in a metaphor. While metaphors are usually signalled by some sort of foregrounding technique, "salience" is not what is most important. Instead, the reason to construe a metaphorical relationship between two elements is that it makes sense to do so; put differently, that such a construal increases overall "relevance" (Sperber and Wilson 1986/1995). Usually, of course, there is a correlation between "salience" and "relevance". In language the identification of two phenomena from different categories (in English often signalled by "is/is like") is a salient feature alerting the recipient to the possibility of a metaphorical construal that achieves relevance by somehow predicting something pertinent about a tenor in terms of a vehicle. But in fact relevance is more important than salience. That is, a hint that metaphorical construal between two elements is a useful strategy may be only marginally salient, but it must result in relevance. Of course in texts (in whatever medium) that have clear communicative purposes (e.g., instructive texts), metaphors are likely to be presented in a strongly salient manner. But in artistic texts (in whatever medium), this is not necessarily the case. In such texts, two elements may be subtly juxtaposed in such a manner that metaphorical construal is invited but not forced (that is, the juxtaposition has low salience) - but achieves relevance (for more discussion see Forceville (1999), where a distinction is proposed between explicitly and implicitly signalled metaphor; and Steen's distinction between "metaphor processing" and “metaphoric processing" (1994: 44f.)).

\section{Can the four main types of pictorial metaphor occur in moving images?}

Above, four subtypes of pictorial metaphor were distinguished. For short, let us label them the contextual type (type A), the hybrid (type B), the simile (type C), and the cross-modal variant (type D). Given that advertisements'and billboards are static images, the metaphorical tenor and vehicle must be recoverable, as it were, in a single glance. Put differently, they must be simultaneously present, either factually (as in types B, C, and D) or virtually (as in type A, wherein one of the terms is not depicted but cued by the pictorial context). In order to further test this fourfold division, however, it will be useful to see how it fares when applied to moving images.

Can the four variants all occur in film? Since all the examples discussed in Forceville (1996) could be imagined as film frames, or freezes, the answer cannot 
but be affirmative. But they are likely to occur in some film genres rather than in others. For one thing, the hybrid type reflects, by definition, a non-natural phenomenon, that is, a phenomenon not encountered as a unity in the world ${ }^{5}$. The constructed character of the hybrid type emphasizes its artificial character. Hybrids are simultaneously two things. This can be suggested in a routine manner by "dressing up" one phenomenon with attributes of another. Indeed, unsubtle comedies make use of characters that are dressed up as, say, animals. Whereas here the "dressing up" can be easily undone, in cartoons the unity between the two elements that are to be construed as tenor and vehicle can be forged in an inextricable fashion, resulting in a single, gestaltlike physical entity. In a more disturbing manner, two phenomena, usually one of them human or humanoid, are turned into a single creature in science fiction and horror movies, with their cyborgs and monsters. Whether, or to what extent, such hybrids are to be considered metaphorically depends on the wider context: in a world consisting of nothing but hybrids, their metaphorical character may disappear (see Kittay 1987: 157).

The cross-modal type also draws attention to itself. Two channels of information each supply the identity of one of the metaphorical terms, thus by definition creating a tension between the information from the two channels. In a silent film, a character shown visually could be verbally announced in an intertitle - a bossy boy could be introduced as "the King" - resulting in the cross-modal BOY IS (LIKE) KING. In the sound film the metaphor's vehicle could be conveyed by another character, saying about the approaching boy, "Here comes the King". Other verbo-pictorial variants are thinkable: a less obtrusive way to convey the metaphor would be to have the bossy boy standing in front of a signpost with the name "King Street" - perhaps with his head concealing the word "street".

The contextual type could be described as a more subtle variant of the hybrid type. Whereas in a hybrid two pictorial elements have been physically conjoined, in the contextual version a pictorial element is metaphorically "transformed" by its context, from which it is, by definition, detached or detachable. The context cues, in the audience, an expectation of how a certain space in the frame is likely to be filled. For this to be possible, the context must be fairly recognizable, even stereotypical. Once such a context has been set up, for a metaphor to be construable expectations must be defied by inserting a different element in that specific space. The result is that the expected element and the "substituted" element can enter into a metaphorical relationship. On the basis of examples in Forceville (1996) my guess is that it is usually the substituted element that will

${ }^{5}$ It may be because of this rather spectacular circumstance that Carroll (1996) considers this type (which he calls a "composite figure" (218)) "core filmic metaphor" (ibid.) while Goldwasser judges it the "most intriguing" subtype (1999: 614) of the four discussed here. For a critical discussion of Carroll (1994, 1996), see Forceville (in press). 
be the tenor of the metaphor, whereas the absent-but-expected element will be its vehicle. To make this rather abstract description more concrete here is an example: if we see a cage in a zoo this context cues the audience into expecting to encounter, in that cage, an animal. If instead we see a man, the suggested metaphor is MAN IS ANIMAL or perhaps MAN IS MONKEY. But the larger context of the film may cause us to reverse this order, or to problematize it. And indeed the overall context, more specifically the film's genre and aims, will help us to decide whether it makes sense to construe a metaphor in the first place - the man could after all simply be a zoo keeper. To distinguish this type from the hybrid type, I argued (Forceville 1996: 163), a literal or imagined stripping away of the context (here: zoo, cage) should destroy the metaphor. From this it can be inferred that some metaphors are intermediate between types. If a man sits crouched, flails his arms, and appears to alternate between munching something and pouting and grinning, no context may be needed to understand that the metaphor MAN IS MONKEY is suggested - in which case this is arguably a metaphor of the hybrid type. But often some form of context (a cage, the branch on which the man sits) will be present as well, so that such a specimen straddles two types.

The simile type, as I defined it, juxtaposes tenor and vehicle in their entirety. A filmic example is a brief shot in Cecil B. DeMille's The Greatest Show on Earth (1952), set in a circus, in which we see a fat man, and then a hippopotamus, suggesting the metaphor FAT MAN IS (LIKE) HIPPOPOTAMUS - although one could here also defend the decision to label this a literal comparison (see Forceville 1996: 142). What matters for this decision is whether one sees fat men and hippopotamuses as belonging to a single superordinate category of FAT CREATURES or whether they are considered as belonging to the categories of HUMANS and ANIMALS respectively.

One aspect in which a pictorial metaphor in cinema is less restricted than a pictorial metaphor in a static picture is that in film, tenor and vehicle need not be presented simultaneously, that is, synchronically. As long as there are signals that two elements must or can be metaphorically coupled, there is no need for mem to co-occur simultaneously. Soviet montage films, notably those by Sergei Eisenstein, provide many examples. A cut from an officer to the statue of a peacock in October (1927-28) suggests the metaphor OFFICER IS PEACOCK, ith the mapped feature, say, of vanity (a more precise meaning depends upon careful examination of the context supplied by the rest of the film). The example of the verbo-pictorial metaphor BOY IS KING in the silent film, given above, also presents tenor and vehicle non-synchronically. This example, incidentally, reveals a second dimension of pictorial metaphor in moving images not available in static ones: the vehicle can temporally precede the tenor. This holds for the cross-modal and the contextual as well as for the simile type. Note that whereas 
the hybrid type at first appears by definition to be possible only in synchronous fashion, this is not necessarily the case. A camera movement may reveal elements of one part of the hybrid before it reveals elements of the other part, so that tenor and vehicle are not synchronically presented.

Since films and commercials have a third important channel of in formation at their disposal besides words and images, namely sound-effects and music, moving images can create metaphors by drawing on the aural channel, too, for the creation of metaphors. Sound effects and music can supply tenors and/or vehicles of metaphors. My intuition is that, at least in narrative films and commercials, sound and music are more likely to yield metaphorical source domains than metaphorical target domains, particularly if they are linked with visual terms. That is, if we see a dog and hear it miaow, there is a greater probability that we are to construe the metaphor DOG IS CAT than CAT IS DOG. This speculation is based on the presupposition that in narratives involving more than one channel of information the visual is dominant over other channels. But it may well be that in non-narrative, experimental films (e.g., in what Bordwell and Thompson (1997: 128) label "associational" and "abstract" films), this dominance is sometimes reversed. An example from a narrative film occurs in Kazan's On the Waterfront (1954), when Terry Malloy (Marlon Brando) confesses to his beloved Edie that he was an accomplice in the murder of her brother. We get alternating shots of Terry and the shocked girl, but while we see his lips move, we cannot hear what he is saying because of a loud boat whistle, also briefly visible, which drowns his words. While the co-occurring of his confession and the whistle can be explained on purely realistic grounds, we may, in addition, construe the metaphor CONFESSION IS (LIKE) WHISTLE, with the feature mapped from vehicle to tenor target being its frightening and alarming nature. (Notice, incidentally, that the audience, who already know of Terry's deed, are made to experience the metaphor from Edie's point of view.)

Just as in language, however, a film may feature not just a very local metaphor, but recurring manifestations of a more deep-rooted, conceptual metaphor. Simons (1995) investigated such conceptual metaphors in a number of television spots in which Dutch political parties promote themselves before elections. Among the spots analyzed there are two ("The Walk", $142 \mathrm{ff}$. and "The Drive", $158 \mathrm{ff}$.) which both draw on the concept of the source domain of a JOURNEY. Roughly speaking, they employ a metaphor that could be verbalized as POLITICAL PROGRESS AS ENVISAGED BY OUR POLITICAL PARTY IS A JOURNEY, and elements in both the visual and the soundtrack contribute to the establishment and elaboration of this metaphor. In similar fashion I have argued in Forceville (1999) that Paul Schrader's film The Comfort of Strangers (like the novel by Ian McEwan on which it is based) in a variety of ways develops the metaphor COLIN IS A CHILD. Both approaches investigate information 
supplied by more than one channel to make a point about an important conceptual metaphor permeating the respective "texts".

\section{Issues for further research}

Let me end by suggesting, in light of the above, a number of questions evoked by nonverbal and cross-modal metaphor, specifically metaphors involving at least one pictorial term. Further examination of these questions will help further our understanding of the nature of metaphor in all its possible manifestations, and provides a fruitful angle for analyzing pictorial communication.

(1) Do the four types of metaphor hitherto identified (contextual, hybrid, simile, crossmodal) exhaust the types or should yet other variants be distinguished? Can these four types be usefully further subdivided? How does Whittock's (1990) typology compare to the one presented here?

(2) Is there any systematic relationship between types of metaphors used and communicative purposes? And between types of metaphors used and specific pictorial (film)genres?

(3) Which cross-modal combinations are possible? If a metaphor is of the cross- modal kind, can correlations be established between the distribution of tenor/ vehicle "slots" on the one hand and the channel of information chosen for each of them on the other?

(4) When do metaphors presenting their two terms non-synchronically supply a source domain before a target domain? What is the effect of this?

(5) How crucial is visual similarity between the two terms of a pictorial metaphor, and to what extent does this vary for the subtypes discussed?

(6) What role does pictorial metonymy play in comparison to pictorial metaphor? What is the relation between pictorial metaphor and pictorial symbolism?

(7) Under what circumstances, if any, can it be argued that tenor and vehicle are reversible in pictorial metaphor? This is a fundamental issue of metaphor studies in general, but it has hitherto not been systematically discussed with regard to visual manifestations. Starting points for discussion can for instance be found in Forceville (1995) and Stockwell (1999).

In order to discuss these questions sensibly, of course, it is first of all imperative that many more examples of pictorial metaphor are identified and analyzed.

\section{Acknowledgments}

I am indebted, as on many previous occasions, to Lachlan Mackenzie (Vrije Universiteit Amsterdam) for his critical comments on an earlier version of this article. 


\section{References}

Aumont, J. (1997). The Image. Trans, by Claire Pajackowska. London: British Film Institute. Originally published as L'Image (1990).

Black, M. (1979). More about metaphor. In: Andrew Ortony (ed.), 19-43.

Bordwell, D., and K. Thompson (1997). Film Art: An Introduction (5th ed.). New York etc.: McGraw-Hill.

Cameron, L., and G. Low (eds) (1999), Researching and Applying Metaphor. Cambridge: Cambridge UP.

Carroll, N. (1994). Visual metaphor. In: Jaakko Hintikka (ed.) Aspects of Metaphor. Dor drecht: Kluwer, 189-218.

Carroll, N. (1996). A note on film metaphor. In: Carroll, Theorizing the Moving Image. Cambridge: Cambridge UP, 212-223.

Forceville, Ch. (1988). The case for pictorial metaphor: Rene Magritte and other Surrealists. In: Ales E. (ed.), Vestnik IMS 9:1. Institut za Marksistićne Śtudije, Ljubljana, 150-160.

Forceville, Ch. (1995) (A)symmetry in metaphor: the importance of extended context. Poetics Today, 16:4, 677-708.

Forceville, Ch. (1996). Pictorial Metaphor in Advertising. London/New York: Routledge. Forceville, Ch. (1999). The metaphor COLIN IS A CHILD in Ian McEwan's, Harold

Pinter's, and Paul Schrader's The Comfort of Strangers. Metaphor and Symbol 14:3, 17998.

Forceville, Ch. (2000). Compasses, beauty queens and other PCs: pictorial metaphors in computer advertisements. Hermes: Journal of Linguistics 24, 31-55.

Forceville, Ch. (in press). The identification of target and source in pictorial metaphors. Journal of Pragmatics.

Goatley, A. (1997). The Language of Metaphors. London/New York: Routledge. Goldwasser, O. (1999). Review of Forceville (1996). Journal of Pragmatics 31, 609-18. Kennedy, X. J., and D. Gioia (1998). An Introduction to Poetry (9th ed.). New York etc.:

Longman.

Kittay, E. F. (1987). Metaphor: Its Cognitive Force and Linguistic Structure. Oxford: Clarendon Press.

Lakoff, G. (1987). Women, Fire and Dangerous Things: What Categories Reveal about the Mind. Chicago: University of Chicago Press.

Lakoff, G., and M. Johnson (1980). Metaphors We Live By. Chicago: U of Chicago P.

Lakoff, G., and M. Turner (1989). More Than Cool Reason: A Field Guide to Poetic Metaphor. Chicago/London: U of Chicago P.

McNeill, D. (1992). Hand and Mind: What Gestures Reveal about Thought. Chicago/ London: University of Chicago Press.

Ortony, A. (ed.) (1979). Metaphor and Thought. Cambridge: CUP. (The second, expanded and revised edition appeared in 1993.)

Satyamurti, C. (1996). Spring offensive. In: Peter Porter and Christopher Hope (eds), New Writing 5, London: Vintage, 371-72. 
Simons, J. (1995). Film, Language, and Conceptual Structures: Thinking Film in the Age of Cognitivism. Unpublished PhD thesis, Dept. of Film and Television Studies, University of Amsterdam.

Sperber, D., and D. Wilson (1986). Relevance: Communication and Cognition. Oxford: Blackwell. (The second, revised edition appeared in 1995.)

Steen, G. (1994). Understanding Metaphor in Literature: An Empirical Approach. London/ New York: Longman.

Stockwell, P. (1999). The inflexibility of invariance. Language and Literature 8:2, 125-42.

Whittock, T. (1990). Metaphor and Film. Cambridge: Cambridge UP.

Zahavi, H. (1993). Dirty Weekend. London: HarperCollins (first published in 1991). 